\title{
Improved Interference Cancelation Channel Estimation Method in OFDM/OQAM System
}

\author{
Shangfei Qiu (D), Lunsheng Xue, and Peng Wu \\ Air and Missile Defense College, Air Force Engineering University, Xian, China \\ Correspondence should be addressed to Shangfei Qiu; qiufei514338459@163.com
}

Received 2 April 2018; Revised 20 October 2018; Accepted 23 October 2018; Published 1 November 2018

Academic Editor: Xuping Zhang

Copyright (C) 2018 Shangfei Qiu et al. This is an open access article distributed under the Creative Commons Attribution License, which permits unrestricted use, distribution, and reproduction in any medium, provided the original work is properly cited.

\begin{abstract}
To the significant amount of pilot overhead of the interference cancelation methods in orthogonal frequency division multiplexing (OFDM) based on offset quadrature amplitude modulation (OFDM/OQAM) system, we proposed an improved interference cancelation method (ICM) for OFDM/OQAM system in this paper. In this method, we use the auxiliary pilot (AP) to eliminate the influence of the intersymbol interference on channel estimation, which can reduce the pilot overhead of OFDM/OQAM system significantly. At the same time, to improve the channel estimation performance, we analyze the source of the intrinsic interference of system and its distribution in time and frequency domain, then, we reset the interference cancelation range of AP, which can cancel more intrinsic interference for OFDM/OQAM system. According to the results of performance analysis, compared to the conventional interference cancelation methods, the proposed method performs better in terms of energy efficiency and spectral efficiency. Also, the simulation results of the proposed method show that the proposed method can outperform traditional interference cancelation methods in channel estimation performance.
\end{abstract}

\section{Introduction}

As an important multicarrier modulation (MCM) scheme, orthogonal frequency division multiplexing (OFDM) based on offset quadrature amplitude modulation (OFDM/OQAM) system, named OQAM system for concision in the rest of the paper, has been proved to outperform OFDM system in the terms of frequency spectrum efficiency, robustness to the interference, and the leakage of the out-band energy [1-4]. However, the performance advantage is obtained at the cost of the relaxation of the orthogonal condition of OQAM system, which only satisfy the orthogonality in the real domain $[5,6]$. This feature causes the intrinsic interference between the real data symbol, which makes the channel estimation method of conventional OFDM system cannot be applied to OQAM system straightforwardly $[7,8]$. Thus, it is necessary to study the new channel estimation method for OQAM system.

At present, the research about channel estimation methods for OQAM system mainly focus on pilots based channel estimation method [9], which can be divided into scattered pilots based [10-15] and preamble-based channel estimation method [16-27]. There are four common scattered pilots based channel estimation methods, zero forcing method [28], auxiliary pilot (AP) method [10], precoding method [11], and iterative method [12]. The AP method set up one dummy symbol in the first order neighborhood of the pilot to cancel the imaginary interference, which was named AP in [13]. This method can cancel mostly interference to the pilot and ensure high spectral efficiency. However, from the view of power efficiency, the AP takes up a significant amount of power.

Preamble-based channel estimation methods have been another major research topic of the exist working, which including the pairs of pilot (POP) method [16], interference approximation method (IAM) [17-19], and the interference cancelation method (ICM) [20-26]. The interference cancelation method eliminates the intercarrier interference (ICI) by utilizing the symmetry of the interference weight coefficient [26]. At the same time, two protective preambles are set on the sides of the pilot preamble to cancel the intersymbol interference (ISI); i.e., there are usually three preambles utilized in the interference cancelation methods. This method can obtain a great channel estimation performance, at the cost of high pilot overhead. The authors in [20-23] proposed three preamble structures for ICM in OQAM system respectively, all of which take up three OQAM real symbols. To reduce 


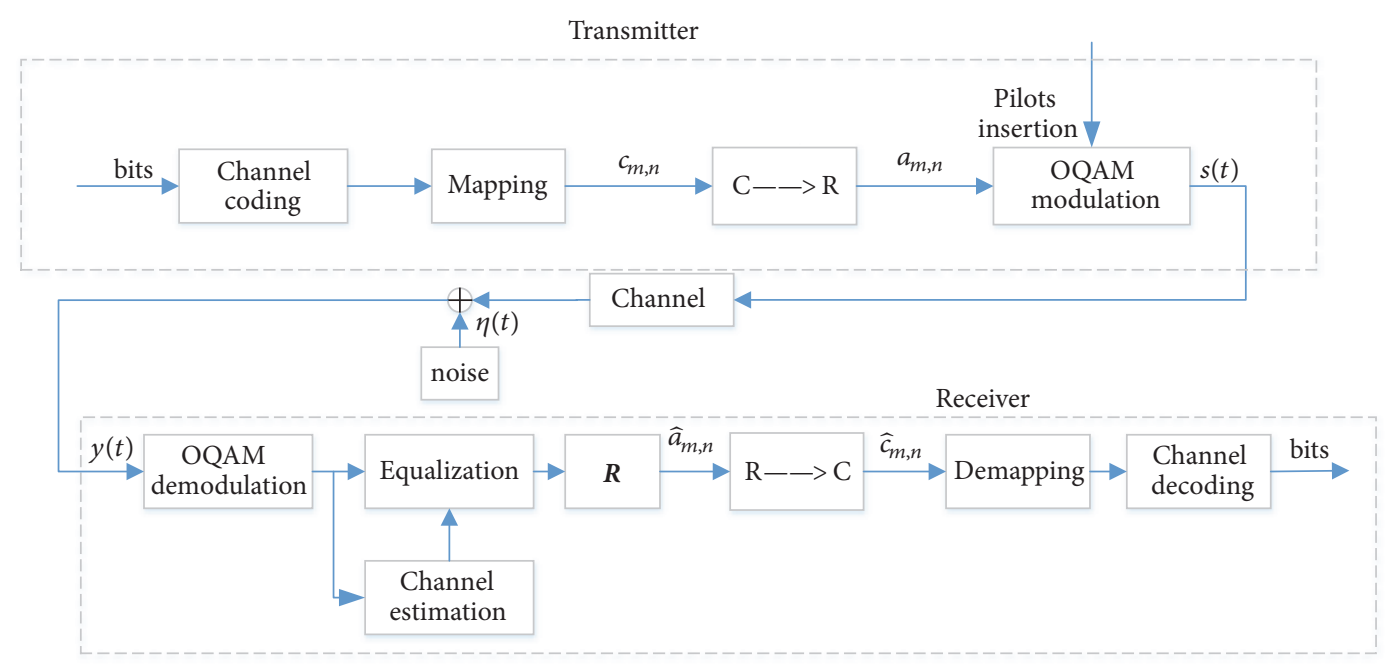

FIGURE 1: OQAM system transmitter and receiver structure.

the high pilot overhead for OQAM system, the author in [24] proposed an iterative interference cancelation method which use only one preamble, with the increase of the computation complexity of system.

From the discussion about the AP method above, it can be known that the AP can reduce the intrinsic interference efficiently. Thus, to the high pilot overhead for the ICM, we utilize the AP instead of the protective preamble to cancel ISI and propose an improved interference cancelation method. At the same time, we take more time frequency points into consideration when eliminating the intrinsic interference, which can improve the channel estimation accuracy.

The remainder of this paper is organized as follows: We have an introduction of OQAM system in Section 2. Then, we provide an overview of the ICM and AP method in Section 3. In Section 4, the proposed method is introduced. Finally, in Section 5, we provide the numerical analysis and simulation result, and we obtain the conclusions in Section 6.

\section{OQAM System Model}

Figure 1 shows the OQAM system transmitter and receiver structure. Firstly, the bits to be sent are channel encode, e.g., using a conventional coder. Then, by QPSK or 16-QAM modulation, the bits are mapped. After the block denoted by $C \longrightarrow R$, the complex symbol $c_{m, n}$ is divided into two real data that correspond to the real and imaginary part of the complex symbol. With the insertion of pilots, the real data $a_{m, n}$ are modulated by the OQAM modulation and transmitted to the receiver by the channel. At the receiver, the received symbol $y(t)$ is the summation of the transmitted symbol $s(t)$ convolved with the channel impulse response and the noise component $\eta(t)$. Firstly, the received symbols are demodulated by OQAM demodulation, and we can obtain the demodulated symbol $y^{\prime}(t)$. Then, through the process of channel estimation, we can get channel estimation value. Combining the channel estimation value and the demodulation symbols $y^{\prime}(t)$, the equalized symbols are derived by the equalization process. Then, by taking the real part of the equalized symbols (denoted by block $R$ ), the real data estimation value $\widehat{a}_{m, n}$ is obtained. Through the block $R \longrightarrow C$ in Figure 1, the complex symbol $\widehat{c}_{m, n}$ is reconstructed from two real data. In the end, the bits are obtained through the demapping and channel decoding process. Now, we briefly describe the channel estimation problem of OQAM system.

We can express the baseband equivalent of a continuoustime OQAM signal as follows [29]:

$$
s(t)=\sum_{m=0}^{M-1} \sum_{n=-\infty}^{+\infty} a_{m, n} \underbrace{\mathrm{e}^{\mathrm{j} \phi_{m, n}} \mathrm{e}^{\mathrm{j} 2 \pi m v_{0} t} g\left(t-n \tau_{0}\right)}_{g_{m, n}(t)}
$$

where $M$ is the even number of subcarrier, $a_{m, n}$ is the real-valued OQAM symbol at the time frequency position $(m, n)$ obtained from a QAM constellation, and $g_{m, n}(t)$ is the prototype filter. The length of filter $g_{m, n}(t)$ is $L_{g}=K M$, and $K$ is the overlapping factor. $v_{0}$ and $\tau_{0}$ denote the subcarrier interval and the time offset between the real and imaginary part of the symbols respectively, and we have $v_{0}=1 / T=$ $1 / 2 \tau_{0}$ with $T$ being the duration of a complex CP-OFDM symbol. The phase term $\phi_{m, n}$, which drives the rule to take the real and imaginary parts, can be expressed as

$$
\phi_{m, n}=\phi_{0}+\frac{\pi}{2}(m+n)
$$

where $\phi_{0}$ can be chosen arbitrarily [19]. Thus, we choose $\phi_{0}=0$ in this paper.

The orthogonality in the real field for OQAM system can be expressed as follows:

$$
\begin{aligned}
\mathfrak{R}\left\{\left\langle g_{m, n} \mid g_{p, q}\right\rangle\right\} & =\mathfrak{R}\left\{\int g_{m, n}(t) g_{p, q}^{*}(t) d t\right\} \\
& =\langle g\rangle_{m, n}^{p, q}=\delta_{m, p} \delta_{n, q}
\end{aligned}
$$

where $\delta$ is the Kronecker delta and $\delta_{m, p}=1$ only when $m=p$; otherwise $\delta_{m, p}=0$. For concision purpose we set $\langle g\rangle_{m, n}^{p, q}=\mathrm{j}\left\langle g_{m, n} \mid g_{p, q}\right\rangle,\left\langle g_{m, n} \mid g_{p, q}\right\rangle$ is a pure imaginary term 


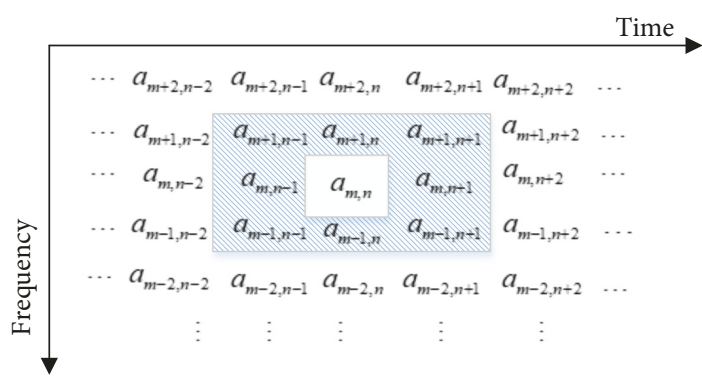

Figure 2: The first order neighborhood for $a_{m, n}$.

when $(m, n) \neq(p, q)$, and we named $\langle g\rangle_{m, n}^{p, q}$ as the interference weight coefficient.

We assume that the OQAM system symbol is transmitted through a channel that varies slowly with time and its delay spread is significantly shorter than the symbol interval. Then, we denote the complex channel gain for the $m$ th subcarrier at the nth symbol interval by $H_{m, n}$. At the same time, we introduce a complex additive white Gaussian noise (AWGN) whose variance is $\sigma^{2}$ at the channel output. Thus, the received symbol at the time and frequency location $(m, n)$ can be obtained as follows [18]:

$$
y_{m, n}=H_{m, n} c_{m, n}+\eta_{m, n}=H_{m, n}\left(a_{m, n}+\mathrm{j} u_{m, n}\right)+\eta_{m, n}
$$

where $\eta_{m, n}$ is the noise term caused by AWGN and $u_{m, n}$ is the intrinsic interference caused by the data symbols surrounding $a_{m, n}$. The intrinsic interference $u_{m, n}$ can be expressed as follows:

$$
u_{m, n}=\mathrm{j} \sum_{(p, q) \in \Omega,(p, q) \neq(m, n)} a_{m, n}\langle g\rangle_{m, n}^{p, q}
$$

where $\Omega$ is the neighborhood for the time and frequency index $(m, n)$, i.e., whose intrinsic interference cannot be ignored. Usually, due to the great time frequency location characteristics of the prototype filter, the first order neighborhood of the pilot is generally considered, which can be shown as the shadow part of Figure 2.

From the discussion above, we can find that the channel estimation for OQAM system cannot as simple as the traditional OFDM system because of the intrinsic interference caused by the neighborhood data symbols. Thus, the channel estimation is one of the major technical challenges in the design of OQAM system.

\section{Interference Cancelation Method and AP Method}

3.1. Interference Cancelation Methods. The spread of the interference weight coefficient of any prototype filter is symmetric [26], which can be shown as follows:

$$
\begin{array}{ccc}
(-1)^{P} \varepsilon & 0 & (-1)^{P} \varepsilon \\
(-1)^{P} \chi & -\beta & (-1)^{P} \chi \\
-(-1)^{P} \kappa & \mathrm{d}_{p, q} & (-1)^{P} \kappa \\
(-1)^{P} \chi & -\beta & (-1)^{P} \chi \\
(-1)^{P} \mathcal{E} & 0 & (-1)^{P} \varepsilon
\end{array}
$$

with the horizontal and vertical direction corresponding to time and frequency, respectively. Above parameters can be computed by equations as follows [26]:

$$
\begin{aligned}
\beta= & e^{-j(2 \pi / M)\left(L_{g}-1\right) / 2} \sum_{l=0}^{L_{g}-1} g^{2}(l) e^{j(2 \pi / M) l} \\
\kappa= & \sum_{l=M / 2}^{L_{g}-1} g(l) g\left(l-\frac{M}{2}\right) \\
\chi= & -j e^{-j(2 \pi / M)\left(L_{g}-1\right) / 2} \\
& \cdot \sum_{l=M / 2}^{L_{g}-1} g(l) g\left(l-\frac{M}{2}\right) e^{j(2 \pi / M) l} \\
\mathcal{E}= & e^{\mp j(2 \pi / M)\left(L_{g}-1\right)} \cdot \sum_{l=M / 2}^{L_{g}-1} g(l) g\left(l-\frac{M}{2}\right) e^{ \pm j 2(2 \pi / M) l}
\end{aligned}
$$

The most direct way to cancel the interference is to simply set zero at the time frequency points around the pilot. From [17], we can find that this idea was already used in the IAM preambles, where protective OQAM symbols were inserted to cancel the interference from preceding and following data. If zeros are transmitted at the middle symbol, i.e., at all oddindexed (or all even-indexed) subcarriers, the interference cancelation can be further simplified. Also, it can maintain a great peak to average ratio (PAPR) performance. This idea can be shown as Figure 3(a), where the channel frequency response (CFR) can be estimated at the even-indexed (oddindexed) subcarriers just like OFDM system. The CFR at the rest of subcarriers can be estimated via interpolation subsequently [20], and it was independently included in the preamble evaluation study of [30] for multiple input multiple output (MIMO) system.

There is a less direct preamble design, which relies in the symmetries in (6) to cancel the interference caused by the adjacent subcarriers. This preamble was proposed in [21] and can be depicted in Figure 3(b).

In [22], a preamble that more replied on the symmetry in (6) was suggested, which canceled both the ISI and ICI of OQAM system by this symmetry. This preamble is shown as Figure 3(c); same as the preamble in Figure 3(b), it can obtain all the CFR without an interpolation process.

Table 1 is a comparison of this three preambles. What is more, to guarantee the channel estimation accuracy, the preamble in Figures 3(b) and 3(c) must satisfy the condition $H_{m-1}=H_{m+1}$; thus, the coherent bandwidth $B_{c}$ is required to meet $3 v_{0} \leq B_{c}$. However, the assumption is hard to guarantee in many conditions [20]. Thus, these two preambles cannot obtain a great ICI robustness of OQAM system.

3.2. AP Method. In general, it only considered the interference coming from the first order neighborhood in the scattered pilot method for OQAM system, for the great time frequency location of the prototype filter. We can set $a_{k}$ as the real data around the pilot at the position $k$ and $\gamma_{k}$ as the interference weight coefficient at the position $k$, where Figure 4 gives an illustration of this notation [12]. 
TABLE 1: Comparison of three preambles.

\begin{tabular}{|c|c|c|c|c|c|c|}
\hline preamble & Pilot overhead & $\begin{array}{c}\text { Interference } \\
\text { cancelation range }\end{array}$ & ISI cancelation & ICI cancelation & $\begin{array}{l}\text { Interpolation } \\
\text { process }\end{array}$ & $\begin{array}{c}\text { PAPR } \\
\text { performance }\end{array}$ \\
\hline ICM1 & $\begin{array}{c}\text { Three OQAM } \\
\text { symbols }\end{array}$ & $\begin{array}{c}\text { First order } \\
\text { neighborhood }\end{array}$ & Zero preamble & Zero symbol data & need & best \\
\hline ICM2 & $\begin{array}{c}\text { Three OQAM } \\
\text { symbols }\end{array}$ & $\begin{array}{c}\text { First order } \\
\text { neighborhood }\end{array}$ & Zero preamble & Coefficient symmetry & No need & moderate \\
\hline ICM3 & $\begin{array}{l}\text { Three OQAM } \\
\text { symbols }\end{array}$ & $\begin{array}{c}\text { First order } \\
\text { neighborhood }\end{array}$ & $\begin{array}{l}\text { Coefficient } \\
\text { symmetry }\end{array}$ & Coefficient symmetry & No need & worst \\
\hline
\end{tabular}

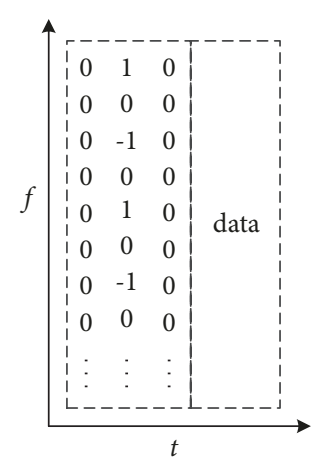

(a) ICM-1

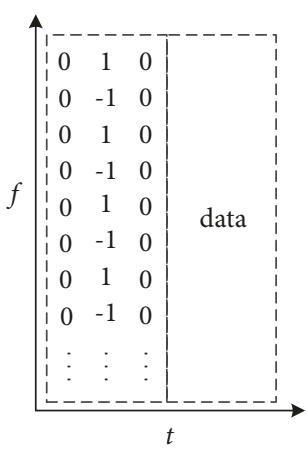

(b) ICM-2

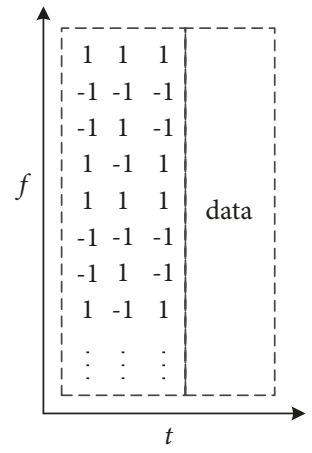

(c) ICM-3

FIgURE 3: Three different preambles for interference cancelation method.

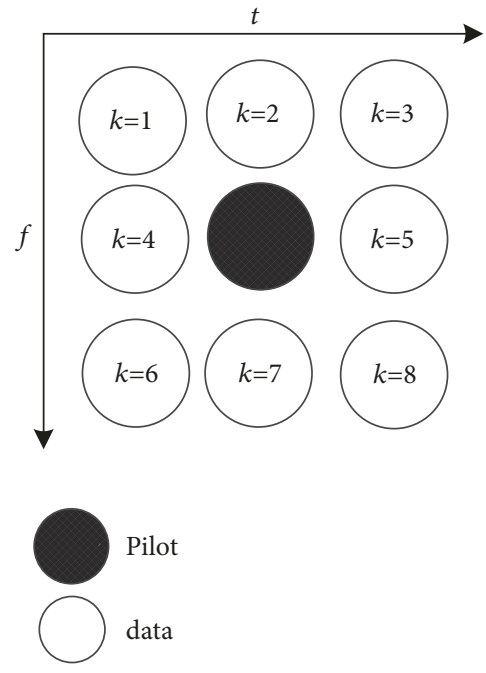

FIGURE 4: Pilot indicating index notation.

The idea of the AP method is to transmit seven time frequency points $i_{1}, i_{2}, i_{3}, i_{4}, i_{5}, i_{6}, i_{7}$ i.e., $a_{i_{k}}=d_{k}$ when $k \in$ $[1, \cdots, 7]$. Then, we can transmit the data that can cancel the interference caused by the other seven data symbols in the first order neighborhood at the position $i_{8}$. And we can draw the equation of AP as follows:

$$
a_{i_{8}}=-\sum_{k=1}^{7} \frac{a_{i_{k}} \gamma_{i_{k}}}{\gamma_{i_{8}}}
$$

Thus, $a_{i_{8}}$ is not really a transmitted data symbol, and this method can obtain a great channel estimation accuracy.
However, there is a major problem with this method that the overall power of the AP. Based on (11), we can draw the average power of AP:

$$
\sigma_{a_{i_{8}}}^{2}=\sigma_{a}^{2} \sum_{k=1}^{7}\left|\frac{\gamma_{i_{k}}}{\gamma_{i_{8}}}\right|^{2}
$$

where $\sigma_{a}^{2}$ is the power of the real data $a_{m, n}$. For example, to the isotropic orthogonal transform algorithm (IOTA) prototype filter with $K=4$, and set $i_{8}=4$, we have

$$
\sigma_{i_{8}}^{2}=4.07 \sigma_{a}^{2}
$$

\section{Proposed Interference Cancelation Method}

Just as the comparison of three preambles in Table 1 shown, all three preambles take up three OQAM symbols, and the high pilot overhead of system is unavoidable, which reduces the practicability of the interference cancelation method. Moreover, those three methods only considered the interference of the first order neighborhood and ignored the interference outside this neighborhood. We must take the interference outside the first order neighborhood into consideration if we want to obtain a better channel estimation performance. Thus, with the interference outside the first neighborhood, we can rewrite (4) as follows:

$$
y_{m, n}=H_{m, n}\left(a_{m, n}+j u_{m, n}^{1}+j u_{m, n}^{2}\right)+\eta_{m, n}
$$


TABLE 2: Interference weight coefficient of IOTA prototype filter.

\begin{tabular}{|c|c|c|c|c|c|c|c|}
\hline \multirow{2}{*}{$f$} & \multicolumn{7}{|c|}{$t$} \\
\hline & -3 & -2 & -1 & $0(\mathrm{n})$ & 1 & 2 & 3 \\
\hline 3 & $4.633 \times 10^{-4}$ & 0.001573 & $0.01027^{*}$ & 0.01824 & $0.01027^{* *}$ & 0.001573 & $4.633 \times 10^{-4}$ \\
\hline 2 & -0.001573 & 0 & $-0.03805^{*}$ & 0 & $0.03805^{* *}$ & 0 & 0.001573 \\
\hline 1 & $0.01027^{*}$ & $0.03805^{*}$ & $0.2280^{*}$ & 0.4411 & $0.2280^{* *}$ & $0.03805^{* *}$ & $0.01027^{* *}$ \\
\hline $0(\mathrm{~m})$ & $-0.01824^{*}$ & 0 & $-0.4411^{\dagger}$ & 1 & $0.4411^{\ddagger}$ & 0 & $0.01824^{* *}$ \\
\hline-1 & $0.01027^{*}$ & $-0.03805^{*}$ & $0.2280^{*}$ & -0.4411 & $0.2280^{* *}$ & $-0.03805^{* *}$ & $0.01027^{* *}$ \\
\hline-2 & -0.001573 & 0 & $-0.03805^{*}$ & 0 & $0.03805^{* *}$ & 0 & 0.001573 \\
\hline-3 & $4.633 \times 10^{-4}$ & -0.001573 & $0.01027^{*}$ & -0.01824 & $0.01027^{* *}$ & -0.001573 & $4.633 \times 10^{-4}$ \\
\hline
\end{tabular}

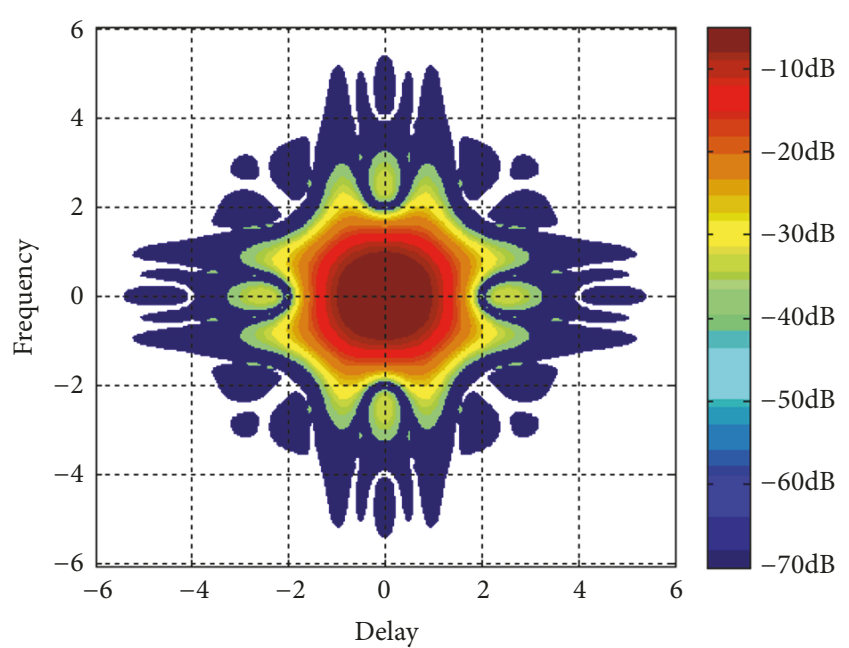

FIGURE 5: Ambiguity function contour line of IOTA prototype filter.

with

$$
\begin{aligned}
& u_{m, n}^{1}=\mathrm{j} \sum_{p, q \in \Omega_{1},(p, q) \neq(m, n)} a_{m, n}\langle g\rangle_{m, n}^{p, q} \\
& u_{m, n}^{2}=\mathrm{j} \sum_{(p, q) \notin \Omega_{1},(p, q) \neq(m, n)} a_{m, n}\langle g\rangle_{m, n}^{p, q}
\end{aligned}
$$

In this paper, we take the IOTA prototype filter [15] as an example to analyze the distribution of the intrinsic imaginary interference in the OQAM system. The interference weight coefficient of IOTA prototype filter is shown in Table 2, and its ambiguity function contour line is depicted in Figure 5. According to both Table 2 and Figure 5, we can see that IOTA filter still has some imaginary part interference outside the first order neighborhood. Ignoring the existence of the intrinsic interference will inevitably limit the performance of the channel estimation. Thus, we take this part of interference into account when designing the preamble of the method in this paper.

Figure 6 is the preamble designed in this paper for the $M=8$ example. Compared to the conventional preambles of interference cancelation method, we only set the APs at the odd-indexed (or even-indexed) subcarriers and can set data symbol at other subcarriers, which can reduce the pilot overhead of the system significantly. And the main idea of the proposed method is introduced in the following.

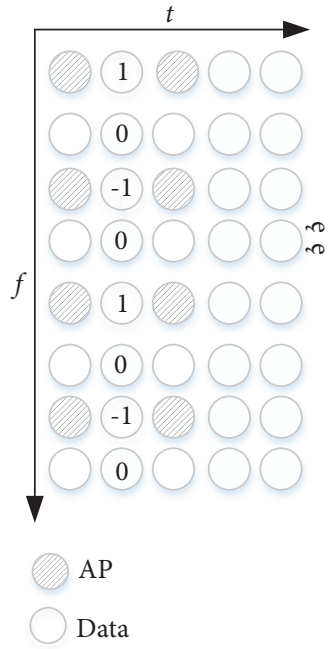

Figure 6: Preamble designed in this paper.

Firstly, to the high pilot overhead of the interference cancelation methods in Section 3, we removed the protective preambles and only retained the middle preamble from which the CFR was recovered. To guarantee the great peak to average ratio (PAPR) performance, we introduced the scheme shown in Figure 3(a).

Secondly, in [15], it is pointed out that the OQAM system intrinsic interference has little effect on the system channel estimation when the interference symbol energy of the pilot is less than $-40 \mathrm{~dB}$. Thus, with the combination of the Figure 3 and Table 2, we mainly eliminate the interference of data symbols transmitted at time frequency points where the interference weight coefficient is larger than 0.01 . It can be covered near the value $-50 \mathrm{~dB}$ in the contour line map of the IOTA prototype filter ambiguity function and can almost completely eliminate the intrinsic imaginary interference in the pilots.

Moreover, according to (11) and (12), we notice that a small absolute value of $\gamma_{i_{8}}$ can result in large magnitude of the AP, which will waste transmission energy and has a bad influence on the OQAM system PAPR performance. Therefore, it is preferable to choose the AP in such a position that the magnitude of the denominator is maximized. Thus, according to the interference weight coefficient in Table 2, we set the AP at the time frequency points preceding 
and following the pilot. Taking two APs of any pilots as example, as depicted in Table 2, the intrinsic interference cancelation range is the special labed time frequency points, “*” corresponds to “ $\dagger$ ”, “**” corresponds to “ $\ddagger$ ”, respectively, and each AP interference cancelation range contains 11 time frequency points. Then, we can draw the equation of the AP in this paper as follows:

$$
a_{m \pm 1, n}=-\sum_{k=1}^{11} \frac{a_{i_{k}} \gamma_{i_{k}}}{\gamma_{m \pm 1, n}}
$$

According to the above description, we can show the preamble designed in this paper in Figure 6 for the $M=8$ example.

\section{Performance Analysis and Simulation Results}

In this section, we compared the performance of the conventional interference cancelation methods with the method proposed in this paper in two aspects of energy efficiency and pilot overhead firstly, and then the performance simulation of the four methods was compared.

5.1. Energy Efficiency Analysis. All the three interference cancelation methods in Section 3 have no additional energy consumption during the transmission process and can maintain stable energy efficiency performance.

For the proposed method in this paper, according to the interference cancelation range and (13), we can express the average power of AP in this paper:

$$
\sigma_{a_{i_{8}}}^{2}=\sigma_{a}^{2} \sum_{k=1}^{11}\left|\frac{\gamma_{i_{k}}}{\gamma_{i_{8}}}\right|^{2}
$$

With the interference weight coefficient of IOTA prototype filter in Table 2 and the position of AP, it is easy to calculate the average power of one AP in this paper:

$$
\sigma_{a_{i 8}}^{2}=0.5680 \sigma_{a}^{2}
$$

Thus, it is clear that the proposed method in this paper does not generate additional energy consumption like the AP method and can achieve a certain increase in energy efficiency.

The energy efficiency improvement of this method is mainly attributed to the mathematical ratio of the interference weight coefficients in different positions of the IOTA prototype filter.

In general, this method is superior to the other three methods in energy efficiency performance, which is attributed to the selection of the AP position and the good time frequency location characteristics of the prototype filter.

5.2. Pilot Overhead Analysis. The analysis of pilot overhead is mainly based on the measurement of the number of time frequency points the preamble occupied. An example of an OQAM system with 2048 subcarriers and 40 OQAM symbols, all the three methods in Section 3 take up 6144 time frequency points. To the method proposed in this paper, the intermediate preamble needs 2048 time frequency points, and the APs on both sides need 2048 time frequency points in total; i.e., this method takes up 4096 time frequency points in total. Thus, the percentage of pilot overhead reduced in this paper is

$$
\varsigma(\%)=\frac{6144-4096}{6144} \times 100 \% \approx 33.3 \%
$$

In general, the conventional interference cancelation methods require 3 OQAM real value symbols to place the preamble, that is, the equivalent of 1.5 OFDM complex symbols. In this paper, the proposed method is equivalent to the need for 2 OQAM symbols to place the preamble, i.e., the equivalent of one OFDM complex symbol. From the above analysis, we can get that this method has $33.3 \%$ performance improvement compared with the conventional interference cancelation method in pilot overhead, which is significant to the practice of the ICM.

5.3. Simulation Results Analysis. The performance of the method proposed in this paper is simulated in this section and is compared with the three conventional interference cancelation methods in Section 3.

The measurement parameters for channel estimation performance for OQAM system mainly include bit error ratio (BER) and normal mean square error (NMSE). BER refers to the ratio of the number of erroneous bits recovered at the receiver to the total bits of system; NMSE is the mathematical expectation of the ratio of the square of the difference between the estimated channel value and the actual channel value to the square of the actual channel value, which can be expressed as follows:

$$
H_{N M S E}=E\left\{\frac{\|\widehat{H}-H\|^{2}}{\|H\|^{2}}\right\}
$$

where $\widehat{H}$ is the estimated CFR value and $H$ is the actual CFR value. In addition, the author in [31] points out that the NMSE performance of channel estimation is related to the energy efficiency and spectral efficiency of the method; thus, the channel estimation NMSE performance is also an important embodiment of the channel estimation method in energy efficiency and spectral efficiency.

In simulation, we choose the IOTA prototype filter with a tap number of 4 , and the sampling frequency is $9.14 \mathrm{MHz}$. The channel coder in simulation is the convolutional channel coding, with $K=7, \mathrm{~g}_{1}=(133)_{o}, \mathrm{~g}_{2}=(171)_{o}$, and coding rate is $1 / 2$.

Firstly, in order to verify the performance of the proposed method with different channel conditions and subcarrier numbers, we selected the typical multipath model channel A and channel B in the IEEE 802.22 standard and the subcarrier numbers 256, 512, and 2048 to carry out the performance simulation. The parameters of the channel A and B in the IEEE 802.22 standard are shown in the Table 3, and the 
TABle 3: Parameters of the channel A and B in the IEEE 802.22 standard.

\begin{tabular}{|c|c|c|}
\hline \multirow{2}{*}{ Channel A } & Path delay $(\mu s)$ & {$\left[\begin{array}{llllll}0 & 2 & 4 & 7 & 11 & 14\end{array}\right]$} \\
\hline & Power gain $(\mathrm{dB})$ & {$\left[\begin{array}{llllll}0 & -7 & -15 & -22 & -24 & -19\end{array}\right]$} \\
\hline \multirow{2}{*}{ Channel B } & Path delay $(\mu s)$ & {$\left[\begin{array}{llllll}-3 & 0 & 2 & 4 & 7 & 11\end{array}\right]$} \\
\hline & Power gain (dB) & {$\left[\begin{array}{llllll}-6 & 0 & -7 & -22 & -16 & -20\end{array}\right]$} \\
\hline
\end{tabular}

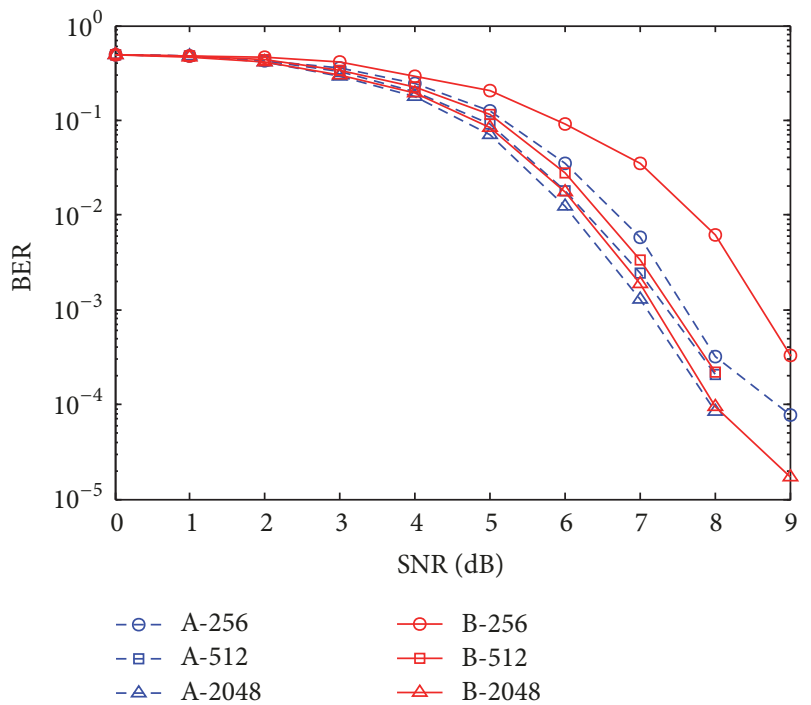

FIGURE 7: BER performance of the proposed method under different channels and subcarrier numbers.

simulation result under different channel and subcarrier numbers is depicted in Figure 7.

From Figure 7, we can see that, under the condition of channel A and channel B, the BER performance of the proposed method is improved with the increase of the number of subcarriers. Meanwhile, under the condition of the same number of subcarriers, the performance of the proposed method under channel $\mathrm{A}$ is better than channel $B$. This is because the average gain of the first path of the channel $B$ is not the maximum gain, which affects the channel estimation performance of the proposed method, but the proposed method can still guarantee a stable channel estimation performance under the channel B. Therefore, the proposed method can ensure stable channel estimation performance with different channel conditions and subcarrier numbers.

Next, we compared the performance of the proposed method under different modulation modes. The channel model used the channel A in the IEEE 802.22 standard. The modulation schemes we selected, QPSK and 16QAM modulation scheme, and the other parameters are consistent with the parameters in the previous simulation, and the simulation result is shown as Figure 8.

Figure 8 is the BER performance comparison of the proposed method between QPSK and 16QAM modulation with different subcarrier numbers. According to the simulation result, we can see that with different subcarrier number, the proposed method can achieve better BER performance in the QPSK modulation mode, and it can obtain about

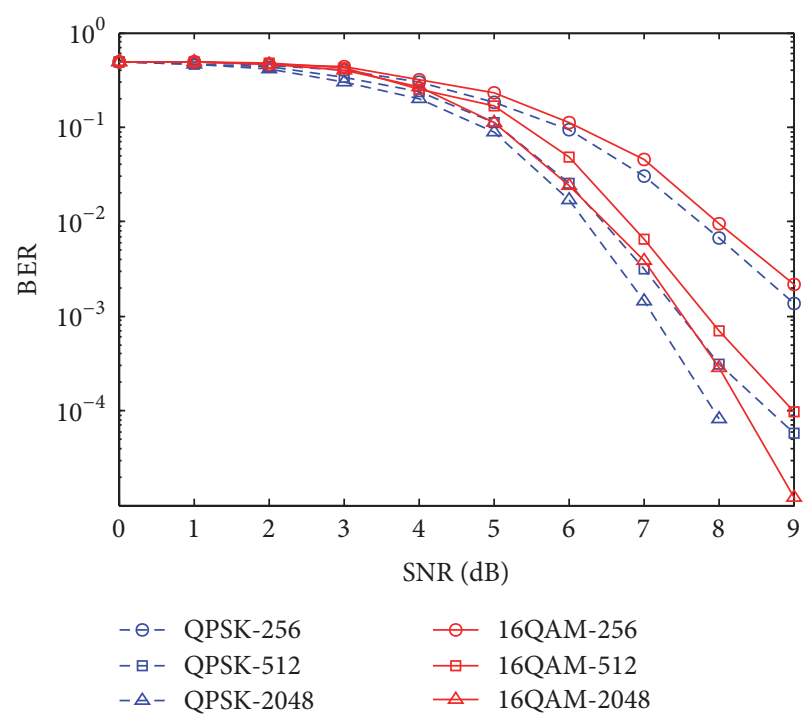

FIGURE 8: BER performance of the proposed method under different modulation modes.

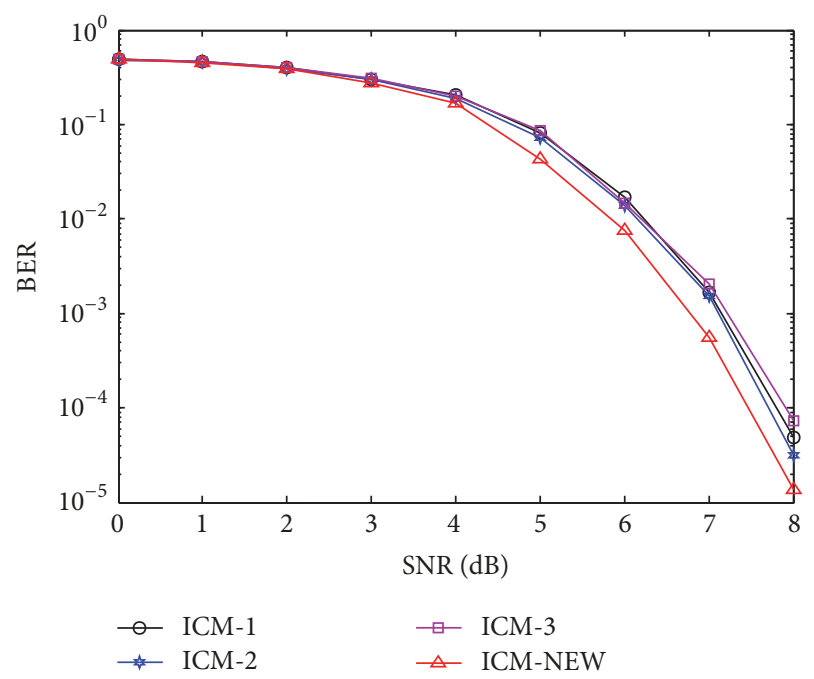

FIGURE 9: BER performance comparison of four methods.

$0.5 \mathrm{~dB}$ performance improvement compared to the 16QAM modulation mode. Overall, the proposed method achieves great BER performance under two modulation modes, which proves the good and stable channel estimation performance of the proposed method.

Finally, we compared the performance of this method with the three interference cancelation methods introduced before. The simulation is based on the channel $\mathrm{A}$, the subcarrier number is 2048, and the modulation mode is QPSK modulation. Meanwhile, in order to avoid the effect of errors caused by different interpolation methods, the proposed method and the traditional method in Figure 3(a) are all use the fast Fourier transform (FFT) interpolation. We used ICM1, ICM-2, and ICM-3 to represent the interference cancelation methods in Figures 3(a), 3(b), and 3(c), respectively, and ICM-NEW represents the method of this paper. Figures 9 and 10 are the performance comparison of BER and NMSE for four methods, respectively. 


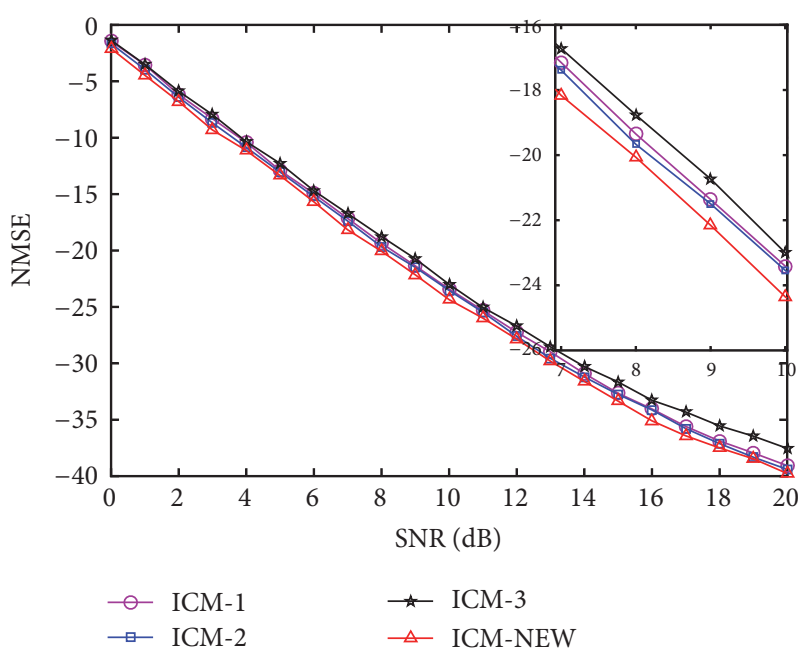

FIGURE 10: NMSE performance comparison of four methods.

From Figure 9, we can see that the proposed method can get better BER performance than the other three methods. As Figure 9 show, the proposed method can get a performance improvement of about $0.4 \mathrm{~dB}$ compared with the ICM-2 method, and it can get about $0.6 \mathrm{~dB}$ performance improvement over ICM-1 and ICM-3.

There are two main points which improved the BER performance of the proposed method. First of all, unlike the conventional interference cancelation methods which only eliminate the intrinsic interference of the first order neighborhood, we enlarged the interference cancelation range. Assuming that all the time frequency points transmit the same real value symbols, compared with the other three methods, the proposed method can eliminate more about $15.8 \%$ intrinsic interference of the OQAM system. Secondly, the proposed method did not reply on the symmetry of the interference weight coefficient to cancel the ISI and ICI, which is important for ICM2 and ICM3. Once the channel bandwidth and the subcarrier interval cannot meet the equation $3 v_{0} \leq B_{c}$, the ICI cancelation accuracy of ICM2 and ICM3 will be greatly reduced. Thus, this is confirmed is Figure 9, where we can see that the proposed method obtain the best BER performance, and ICM3 is the worst one.

With the simulation results of Figure 10, we can see that the proposed method can get better NMSE performance than the other three methods. Although all the four methods obtain similar NMSE performance, the proposed method is still better than other three interference cancelation methods at least $0.2 \mathrm{~dB}$. In (19), we have proved that the proposed method can improved the power efficiency. Also, in (20) we have proved that the spectral efficiency can be further improved by the proposed method. Thus, it was confirmed in Figure 10, where we can that the proposed method obtain a better NMSE performance than other three methods. Besides, this method eliminated more intrinsic interference of the system by enlarging the interference cancelation range, which is also one of the reasons that this method can achieve better NMSE performance.
From the performance analysis and simulation results of the proposed method and the other three interference cancelation methods, we can see that the proposed method is superior to the other three methods in various performance aspects. While reducing the pilot overhead for OQAM system, by increasing the elimination range of the intrinsic interference, the proposed method greatly improves the channel estimation performance for OQAM system.

\section{Conclusion}

To the high pilot overhead of the interference cancelation method in OQAM system, this paper proposed an improved interference cancelation method. In this method, we utilize APs instead of the protective preambles in the conventional interference cancelation methods to cancel the ISI for the recovered preamble, which can significantly reduce the pilot overhead of system. Meanwhile, by increasing the elimination range of the intrinsic interference for system, the channel estimation accuracy of the system is effectively improved. The performance analysis and simulation results of the proposed method show that the proposed method is superior to the previous interference cancelation channel estimation method in terms of energy efficiency, spectral efficiency, and channel estimation performance. Therefore, the method proposed in this paper has a better complex performance and is a more practical channel estimation method for OQAM system.

\section{Data Availability}

The system simulation data used to support the findings of this study are included within the article.

\section{Conflicts of Interest}

The authors declare that there are no conflicts of interest regarding the publication of this paper.

\section{Acknowledgments}

The work of this paper was supported by National Natural Science Fund of China under Grant no. 6167148.

\section{References}

[1] P. Siohan, C. Siclet, and N. Lacaille, "Analysis and design of OFDM/OQAM systems based on filterbank theory," IEEE Transactions on Signal Processing, vol. 50, no. 5, pp. 1170-1183, 2002.

[2] A. Skrzypczak, P. Siohan, N. Chotkan, and M. Djoko-Kouam, "OFDM/OQAM: An appropriate modulation scheme for an optimal use of the spectrum," in Proceedings of the 2008 3rd International Symposium on Communications, Control, and Signal Processing, ISCCSP2008, pp. 405-410, IEEE, St Julians, Malta, March 2008.

[3] D. Katselis, E. Kofidis, A. Rontogiannis, and S. Theodoridis, "Preamble-based channel estimation for CP-OFDM and OFDM/OQAM systems: a comparative study," IEEE Transactions on Signal Processing, vol. 58, no. 5, pp. 2911-2916, 2010. 
[4] D. Vučić, S. Vukotić, and M. Erić, "Cyclic spectral analysis of OFDM/OQAM signals," AEUE-International Journal of Electronics and Communications, vol. 73, pp. 139-143, 2017.

[5] A. Skrzypczak, J. Palicot, and P. Siohan, "OFDM/OQAM modulation for efficient dynamic spectrum access," International Journal of Communication Networks and Distributed Systems, vol. 8, no. 3-4, pp. 247-266, 2012.

[6] Z. Wang and G. B. Giannakis, "Wireless multicarrier communications," IEEE Signal Processing Magazine, vol. 17, no. 3, pp. 29-48, 2000.

[7] S. Vukotic and D. Vucic, "Detection and clasiffication of OFDM/ QAM and OFDM/OQAM signals based on cyclostationary features," in Proceedings of the 23rd Telecommunications Forum, TELFOR 2015, pp. 232-235, IEEE, Belgrade, Serbia, November 2015.

[8] H. Lin and P. Siohan, "Robust channel estimation for OFDM/ OQAM," IEEE Communications Letters, vol. 13, no. 10, pp. 724726, 2009.

[9] R. Barrera, J. Perez, and C. Salazar, "Carbones colombianos: clasificación y caracterización termoquímica para aplicaciones energéticas," Ion, vol. 27, no. 2, 2015.

[10] J. Javaudin, D. Lacroix, and A. Rouxel, "Pilot-aided channel estimation for OFDM/OQAM," in Proceedings of the 57th IEEE Semiannual Vehicular Technology Conference. VTC 2003, pp. 1581-1585, Jeju, South Korea.

[11] C. Lélé, R. Legouable, and P. Siohan, "Channel estimation with scattered pilots in OFDM/OQAM," in Proceedings of the IEEE 9th Workshop on Signal Processing Advances in Wireless Communications (SPAWC '08), pp. 286-290, Recife, Brazil, July 2008.

[12] C. Lélé, "Iterative scattered-based channel estimation method for OFDM/OQAM," EURASIP Journal on Advances in Signal Processing, vol. 2012, no. 1, pp. 1-14, 2012.

[13] T. H. Stitz, T. Ihalainen, A. Viholainen, and M. Renfors, "Pilot-based synchronization and equalization in filter bank multicarrier communications," EURASIP Journal on Advances in Signal Processing, vol. 2010, no. 1, pp. 1-18, 2010.

[14] R. Nissel and M. Rupp, "On pilot-symbol aided channel estimation in FBMC-OQAM," in Proceedings of the 41st IEEE International Conference on Acoustics, Speech and Signal Processing, ICASSP 2016, pp. 3681-3685, IEEE, Shanghai, China, March 2016.

[15] W. Cui, D. Qu, T. Jiang, and B. Farhang-Boroujeny, "Coded Auxiliary Pilots for Channel Estimation in FBMC-OQAM Systems," IEEE Transactions on Vehicular Technology, vol. 65, no. 5, pp. 2936-2946, 2016.

[16] C. Lélé, J.-P. Javaudin, R. Legouable, A. Skrzypczak, and P. Siohan, "Channel estimation methods for preamble-based OFDM/OQAM modulations," European Transactions on Telecommunications, vol. 19, no. 7, pp. 741-750, 2010.

[17] C. Lélé, P. Siohan, and R. Legouable, "2 dB Better Than CPOFDM with OFDM/OQAM for Preamble-Based Channel Estimation," in Proceedings of the IEEE International Conference on Communications, ICC 2008, pp. 1302-1306, IEEE, Beijing, China, May 2008.

[18] C. Lele, "OFDM/OQAM Modulation: Channel Estimation Methods [Ph.D. thesis]," CNAM, 2008.

[19] J. Du and S. Signell, "Novel Preamble-Based Channel Estimation for OFDM/OQAM Systems," in Proceedings of the IEEE International Conference on Communications, ICC2009, pp. 4135-4140, IEEE, Dresden, Germany, June 2009.
[20] S. Hu, G. Wu, T. Li, Y. Xiao, and S. Li, "Preamble design with ICI cancellation for channel estimation in OFDM/OQAM system," IEICE Transactions on Communications, vol. E93-B, no. 1, pp. 211-214, 2010.

[21] S. Kang and K. Chang, "A novel channel estimation scheme for OFDM/OQAM-IOTA system," ETRI Journal, vol. 29, no. 4, pp. 430-436, 2007.

[22] T. Yoon, S. Im, S. Hwang, and H. Choi, "Pilot Structure for High Data Rate in OFDM/OQAM-IOTA System," in Proceedings of the 2008 IEEE 68th Vehicular Technology Conference (VTC 2008-Fall), pp. 1-5, IEEE, Calgary, Canada, September 2008.

[23] F. Deng, X. He, G. Cheng, and S. Li, "An Effective PreambleBased Channel Estimation Structure for OFDM/OQAM Systems," in Proceedings of the 2010 6th International Conference on Wireless Communications, Networking and Mobile Computing (WiCOM), pp. 1-4, Chengdu, China, September 2010.

[24] S. Hu, G. Wu, and S. Li, "Preamble design and iterative channel estimation for OFDM/offset QAM system," Journal of Networks, vol. 4, no. 10, pp. 1050-1057, 2009.

[25] Y. Zhao, X. Chen, L. Xue, J. Liu, and Z. Xie, "Iterative preamblebased time domain channel estimation for OFDM/OQAM systems," IEICE Transactions on Communications, vol. E99B, no. 10, pp. 2221-2227, 2016.

[26] E. Kofidis, D. Katselis, A. Rontogiannis, and S. Theodoridis, "Preamble-based channel estimation in OFDM/OQAM systems: A review," Signal Processing, vol. 93, no. 7, pp. 2038-2054, 2013.

[27] L. Caro, V. Savaux, D. Boiteau, M. Djoko-Kouam, and Y. Louet, "Preamble-based LMMSE channel estimation in OFDM/ OQAM modulation," in Proceedings of the 81st IEEE Vehicular Technology Conference, VTC Spring 2015, pp. 1-5, IEEE, Glasgow, UK, May 2015.

[28] D. Lacroix and J. P. Javaudin, "A new channel estimation method for OFDM/OQAM," in Proceedings of the 7th International OFDM-Workshop, Technical University, Department of Telecommunications, Hamburg, Germany, 2002.

[29] H. Bölcskei, "Orthogonal Frequency Division Multiplexing Based on Offset QAM," in Advances in Gabor Analysis, pp. 321352, Birkhäuser, Boston, MA, USA, 2003.

[30] M. Siyau, T. Li, J. Prieto, J. Corchado, and J. Bajo, "A Novel Pilot Expansion Approach for MIMO Channel Estimation and Tracking," in Proceedings of the IEEE International Conference on Ubiquitous Wireless Broadband, ICUWB 2015, IEEE, Montreal, QC, Canada, October 2015.

[31] J.-M. Choi, Y. Oh, H. Lee, and J.-S. Seo, "Pilot-Aided Channel Estimation Utilizing Intrinsic Interference for FBMC/OQAM Systems," IEEE Transactions on Broadcasting, vol. 63, no. 4, pp. 644-655, 2017. 


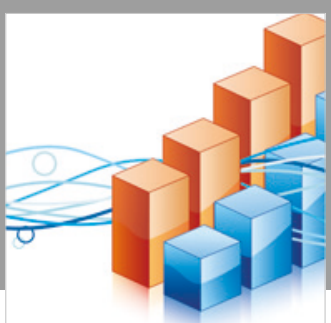

Advances in

Operations Research

\section{-n-m}
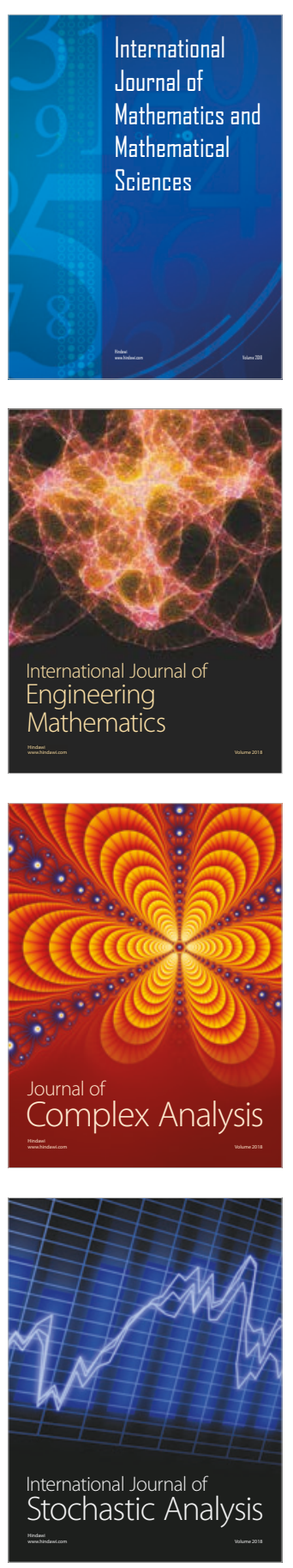
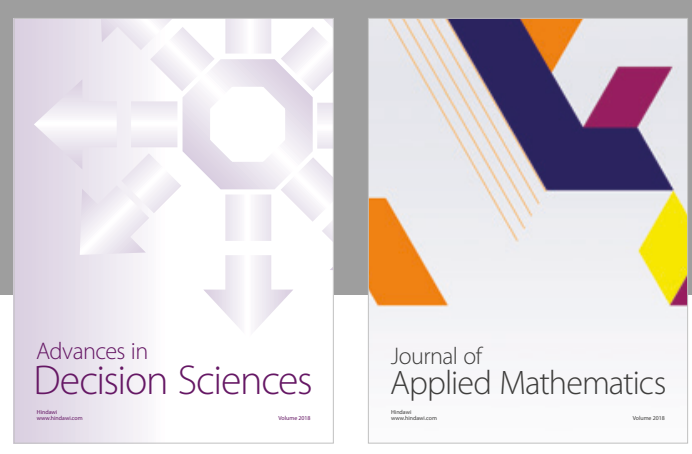

Journal of

Applied Mathematics
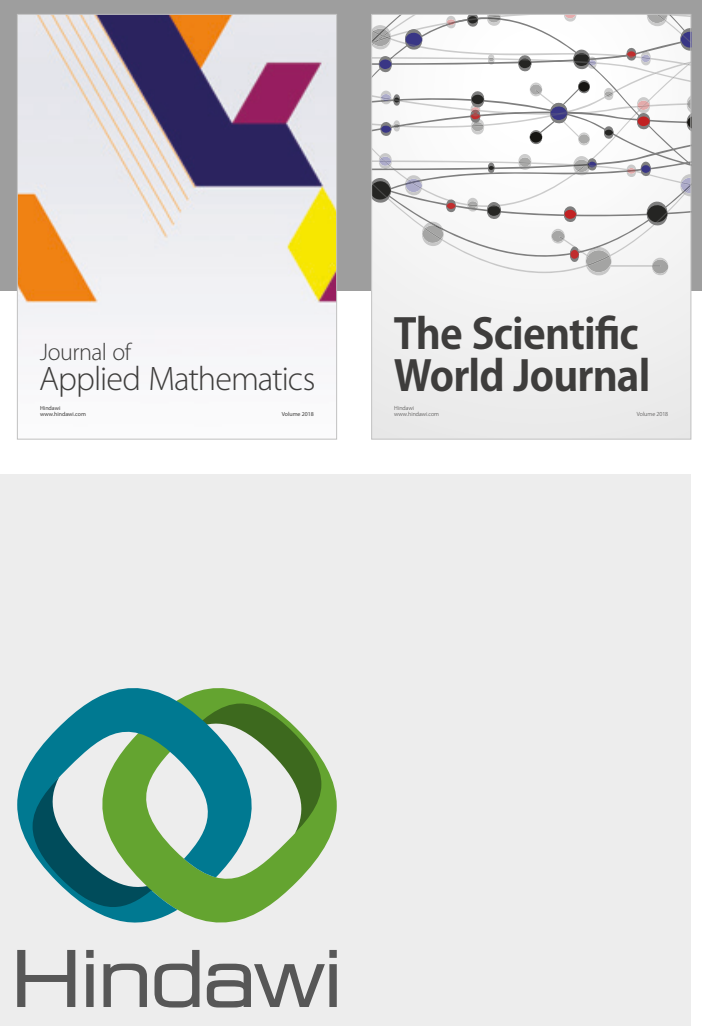

Submit your manuscripts at

www.hindawi.com

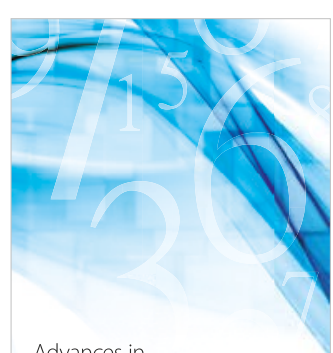

Advances in
Numerical Analysis
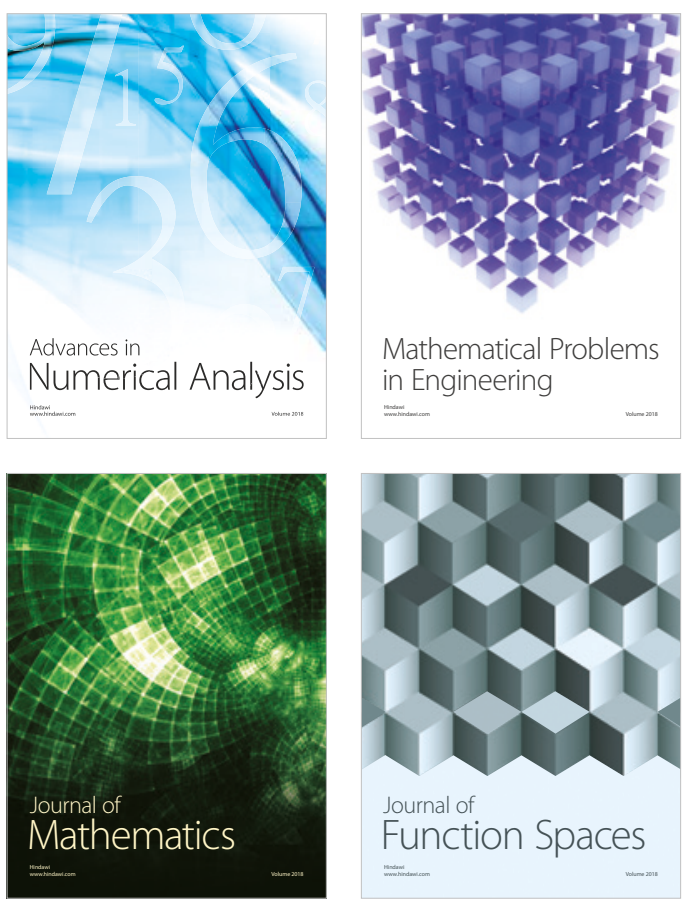

Mathematical Problems in Engineering

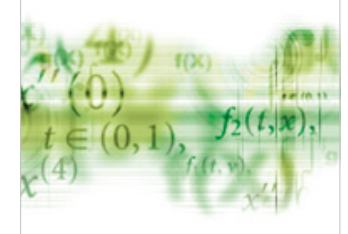

International Journal of

Differential Equations

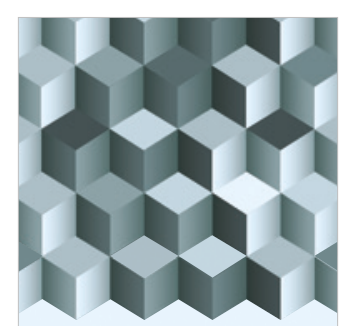

Journal of

Function Spaces

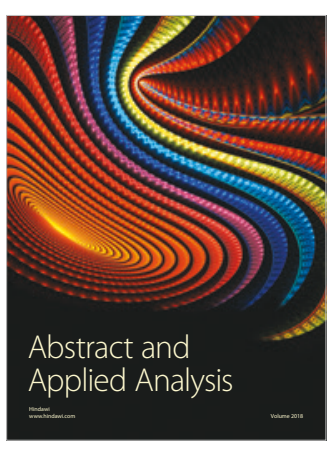

The Scientific

World Journal

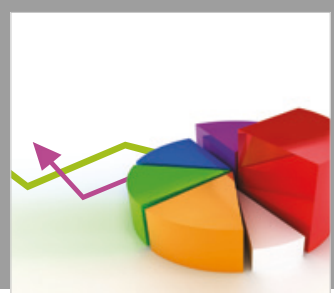

Journal of

Probability and Statistics
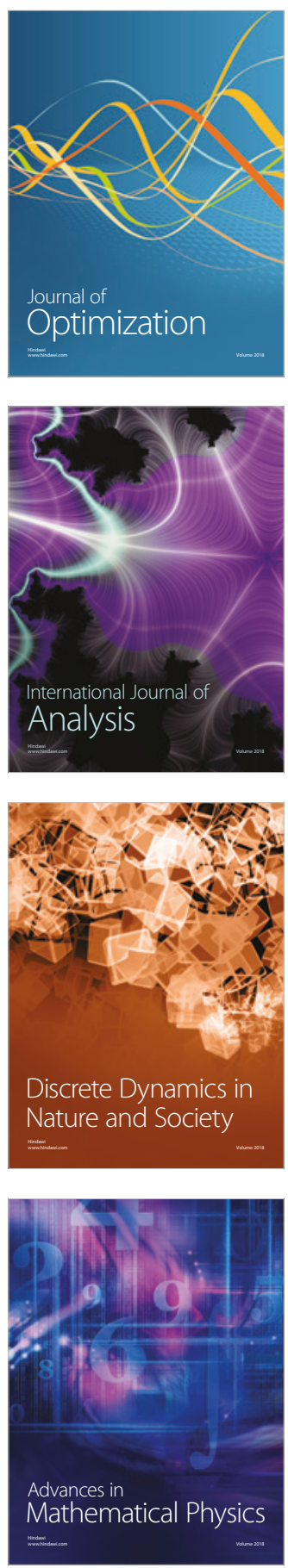